\title{
Unique phenotypes and variation in the sex comb patterns and their evolutionary implications in the Drosophila bipectinata species complex (Diptera: Drosophilidae)
}

\author{
PARAS K. MISHRA and BASHISTH N. SINGH*
}

\author{
Genetics Laboratory, Department of Zoology, Banaras Hindu University, 221005 - Varanasi, India; \\ e-mails: bashisthsingh2004@rediffmail.com; bnsingh@bhu.ac.in
}

\begin{abstract}
Key words. Diptera, Drosophilidae, Drosophila bipectinata species complex, sex comb pattern, interspecific hybridization, phylogenetic relationship
\end{abstract}

\begin{abstract}
Understanding the genetic mechanisms of morphological evolution is one of the greatest challenges in evolutionary biology and for such studies sexually dimorphic traits in closely related species are of prime interest. In the Drosophila bipectinata species complex, which consists of four closely related species, namely D. bipectinata, D. parabipectinata, D. malerkotliana and D. pseudoananassae, the pattern of sex combs (a sexually dimorphic trait) is found to be highly diversified. The present investigation documents some unique and new sex comb phenotypes and demarcates intra- and interspecific variations in the sex comb pattern among the four species and their hybrids. There is remarkable similarity in sex comb pattern of D. bipectinata and D. parabipectinata but it differs from that of $D$. malerkotliana and D. pseudoananassae, which is in consistent with the phylogenetic relationships among the four species traced out by cytological, biochemical and molecular studies. The genetic basis of inheritance of sex comb patterns, its plausible implication with biogeographical distribution of species and the relationship between degree of hybridization and phylogenetic proximity have been addressed.
\end{abstract}

\section{INTRODUCTION}

Sex combs are one of the most striking morphological traits of taxonomic importance that is confined to the subgenus Sophophora under the genus Drosophila. They consist of a row of stout, rounded black bristles (teeth) on the foretarsi (first and second tarsomeres) of males (Lachaise \& Chassagnard, 2000) and exhibit great divergence among the species of Drosophila. Sex comb patterns are a species-specific trait that show considerable numerical variation in teeth among the individuals of the same species. It is a very important morphological feature in differentiating especially those species where other taxonomic characters are either absent or less prominent. In the $D$. bipectinata complex that belongs to the ananassae subgroup of the large and diversified melanogaster species group (subgenus Sophophora, genus Drosophila), there are four closely related species, namely D. bipectinata Duda, 1923, D. parabipectinata Bock, 1971, D. malerkotliana Parshad \& Paika, 1964 and D. pseudoananassae Bock, 1971 (Bock \& Wheeler, 1972; Mishra \& Singh, 2005a). The most obvious phenotypic features for distinguishing these species are sex comb patterns and abdominal tip pigmentation in males (Kopp \& Barmina, 2005). Recently, Matsuda et al. (2005) have described three subspecies of D. bipectinata based on their reproductive isolation, namely $D . b$. bipectinata from Southeast Asia and Okinawa, D. b. szentivanii from Papua New Guinea and D. b. pacificiae from South Pacific Ocean. Bock (1971a) described two subspecies of
D. malerkotliana (D. m. malerkotliana and D. m. pallidalater on named as $D$. m. pallens by Bock \& Wheeler, 1972 ) and D. pseudoananassae (D. p. pseudoananassae and $D$. p. nigrens) on the basis of differences in the male abdominal coloration. In both species, western populations have black abdominal tips while eastern populations have pale brown abdominal tips (Tomimura et al., 2005 and the reference map there in). Males of D. m. malerkotliana have a black abdominal tip and the species is distributed in India, Thailand, Malaya, Java, Sumatra and Africa, while males of $D . m$. pallens have a brown abdominal tip and the species is distributed in Borneo, the Philippines, and Celebes, east of Wallace's line, but not of Weber's line (Okada, 1981; Tomimura et al., 2005). D. p. pseudoananassae is distributed in Australia, New Guinea, the Philippines and Lombok, and the abdominal tip of males is brown, while $D$. p. nigrens is distributed in Sri Lanka, India, Java, Borneo and Malaya, and the abdominal tip of males is black (Okada, 1981; Tomimura et al., 2005). Thus, D. parabipectinata, D. m. malerkotliana and $D$. p. pseudoananassae are pigmented in a sexually dimorphic pattern where the last three abdominal segments are completely melanized in males but not in females (Kopp \& Barmina, 2005). Hence, distinguishing males on the basis of melanization of the abdominal tip may lead to committing mistakes, as there may be very little difference in the density of pigmentation. On the other hand, sex comb morphology is a very prominent and easily distinguishable trait even for a non-

\footnotetext{
* Corresponding author.
} 
taxonomist. Therefore, the study of sex comb phenotypes becomes indispensable to identify correctly and distinguish these four species. However, females of all species and subspecies of this complex are extremely similar and cannot be differentiated morphologically (Bock, 1971a; 1978; Singh \& Singh, 2001; Mishra \& Singh, 2006).

Members of the $D$. bipectinata complex are distributed from east Africa, through Southeast Asia, Australia, India, New Guinea, and the South Pacific Islands, to South America (Tomimura et al., 2005; Kopp \& Barmina, 2005, for details see distribution maps there in). The most widespread among them is D. bipectinata (Bock, 1978; Singh \& Singh, 2001), which is supposed to increasing its distribution range rapidly (Yang et al., 1972).

The four species of this complex are intercrossable in the laboratory but not in natural condition. There is only one report of natural hybridization between $D$. bipectinata and $D$. malerkotliana, although the frequency of hybridization was very low (Gupta et al., 1980). Hybrid males are sterile while females are fertile, which conforms to most interspecific hybrids in the genus Drosophila (Mishra \& Singh, 2005b). The causes of hybrid male sterility have been documented in different interspecific crosses of the D. bipectinata complex (Mishra \& Singh, 2005a, 2006). Further, this complex is emerging as a promising group for investigating phenotypic evolution and speciation (Kopp \& Barmina, 2005). The extensive morphological variation within and among species with respect to the sex comb teeth number and abdominal tip pigmentation, together with the ease of interspecific hybridization and extremely low (283000-385000 years ago) genetic divergence time (Kopp \& Barmina, 2005), makes the $D$. bipectinata species complex an excellent model for investigating the genetic basis of morphological evolution.

Since there is considerable numerical variation in sex comb teeth among the four species, in this communication, we endeavor to make a lucid demarcation of sex comb patterns in each species and their hybrids. Further, the degree of hybridization among the species plausibly reflects their phylogenetic proximity. The other questions addressed are: (1) what is the genetic basis of sex comb inheritance, (2) how sex comb pattern is concerned with the phylogenetic relationship of the four species, and (3) how the variation in the sex comb pattern is correlated with the geographical distribution of these species.

\section{MATERIAL AND METHODS}

The details of the strains used of four members of the $D$. bipectinata complex are given in Table 1. All stocks were maintained in the laboratory on simple yeast-agar culture medium at approximately $24^{\circ} \mathrm{C}$. For scoring sex comb teeth number, 100 males were randomly taken from each stock and the teeth number on right foreleg in each male was scored under a microscope at $100 \times$ magnification after dissecting the forelegs on a slide, mounted with few drops of $0.67 \%$ saline. For hybridization, reciprocal crosses were made among four species by employing one strain of each species $(D . b$. bipectinata $\mathrm{PN}, D$. parabipectina $\mathrm{MY}, D$. m. malerkotliana $\mathrm{RC}$ and $D$. p. nigrens $\mathrm{CM})$. In total, twelve crosses were made by confining 3-4 virgin females with $3-4$ virgin males in a food vial $\left(1^{\prime \prime} \times 3^{\prime \prime}\right)$ to produce
TABLE 1. Details of different strains of all the four species of the D. bipectinata complex.

\begin{tabular}{lcc}
\hline \multirow{2}{*}{ Species } & \multicolumn{2}{c}{ Strains } \\
\cline { 2 - 3 } D. b. bipectinata & TD & Thiruvananthapuram, Kerala, India \\
& NG & Niligiri, Tamilnadu, India \\
& AD & Alipurdwar, West Bengal, India \\
& AR & Arumanai, Tamilnadu, India \\
& MY & Mysore, Karnataka, India \\
& SL & Siliguri, West Bengal, India \\
& PN & Pune, Maharashtra, India \\
D. parabipectinata & MY & Mysore, Karnataka, India \\
& CL & Celebes, Indonesia \\
D. m. malerkotliana & RC & Raichur, Karnataka, India \\
& BP & Baripada, Orissa, India \\
& MY & Mysore, Karnataka, India \\
& BHU & Varanasi, Uttar Pradesh, India \\
& CM & Chiang Mai, Thailand \\
\hline
\end{tabular}

$F_{1}$ hybrids. The teeth number was scored in hundred males in each cross using the same method as mentioned above.

The range of teeth number in each row of first and second tarsomeres of foretarsus, their total number and mean number are calculated in all the four species and their hybrids. To analyse the data one-way analysis of variance (ANOVA), Duncan's multiple-range test (DMRT), t-test and Homogeneity of coefficient of variation have been employed (Zar, 2005).

\section{RESULTS}

Each row of teeth on tarsomeres is called a sex comb. The details of number of teeth, their range of variation in each comb and mean number of teeth for all the strains of four species are given in Table 2. There are two combs on the first tarsomere and one comb on the second tarsomere in $D . b$. bipectinata and they show extreme similarity in pattern with that of $D$. parabipectinata in all the above measurements.

There is large variation in sex comb patterns within $D$. b. bipectinata (Figs 1a-i) in comparison with D. parabipectinata (Table 2B). We found the following new sex comb phenotypes in D. bipectinata: 1 . presence of three sex combs on the first tarsomere (Fig. 1e), 2. some unique phenotypes of sex comb (Figs 1d, f, g, h, i), 3. presence of three teeth in the comb on second tarsomere (Fig. 1c), and 4. absence of sex comb on second tarsomere (Fig. 1b). In addition, another interesting observation documented for the first time is that, instead of a single set of proximal and distal combs on the first and second tarsomeres, there can be two sets, each showing variation in the number of teeth. However, their frequency is less than $15 \%$. For example, in $D$. bipectinata, instead of a single proximal comb set in the TD strain, there are two sets with a number of teeth $6 / 3,4 / 4,5 / 2,3 / 3,2 / 5$ and $4 / 3$ (upper set/lower set). Similarly, in the AR strain, it is $1 / 5$, $1 / 6,1 / 7$ and $7 / 4$; in the MY strain, it is $3 / 1,3 / 3,1 / 7$ and $5 / 1$; in the NG strain, it is $1 / 5,1 / 6,1 / 7,2 / 4,3 / 5,4 / 1,4 / 3$, $5 / 3,6 / 2$ and $7 / 2$; in the AD strain, it is $1 / 4,1 / 5,1 / 6,2 / 5$, $4 / 1,5 / 2$ and $5 / 3$; and in the SL strain, it is $5 / 2$ and $1 / 5$. In 
TABLE 2A. Details of sex comb teeth arrangements in different strains of four species of the D. bipectinata complex.

\begin{tabular}{|c|c|c|c|c|c|c|}
\hline \multirow{2}{*}{ Species } & \multirow{2}{*}{ Strains } & \multicolumn{2}{|c|}{ First tarsomere teeth no.(range) } & \multicolumn{2}{|c|}{ Second tarsomere teeth no. (range) } & \multirow{2}{*}{ Mean $\pm \mathrm{SE}$ (range) } \\
\hline & & Proximal comb & Distal comb & Proximal comb & Distal comb & \\
\hline \multirow[t]{8}{*}{ D. b. bipectinata } & TD & $5(4-7)$ & $7(6-10)$ & $2(1-2)$ & 0 & $15.93 \pm 0.15(12-20)$ \\
\hline & NG & $5(4-7)$ & $8(6-10)$ & $2(1-3)$ & 0 & $15.73 \pm 0.15(13-19)$ \\
\hline & $\mathrm{AD}$ & $5(4-7)$ & $7(5-9)$ & $1(1-2)$ & 0 & $13.54 \pm 0.14(11-17)$ \\
\hline & $\mathrm{AR}$ & $6(5-8)$ & $8(6-10)$ & $1(1-2)$ & 0 & $15.69 \pm 0.14(13-19)$ \\
\hline & MY & $5(4-7)$ & $7(6-10)$ & $1(1-2)$ & 0 & $14.24 \pm 0.14(11-18)$ \\
\hline & SL & $5(4-8)$ & $7(6-10)$ & $1(1-2)$ & 0 & $14.19 \pm 0.18(11-19)$ \\
\hline & $\mathrm{PN}$ & $5(4-7)$ & $7(6-10)$ & $2(0-2)$ & 0 & $14.02 \pm 0.18(10-18)$ \\
\hline & & & & & Mean & $14.75 \pm 0.06$ \\
\hline \multirow[t]{3}{*}{ D. parabipectinata } & MY & $5(4-7)$ & $6(4-10)$ & $2(0-2)$ & 0 & $14.19 \pm 0.19(10-18)$ \\
\hline & $\mathrm{CL}$ & $6(4-8)$ & $8(7-10)$ & $1(0-2)$ & 0 & $15.11 \pm 0.16(11-18)$ \\
\hline & & & & & Mean & $14.65 \pm 0.12$ \\
\hline \multirow[t]{5}{*}{ D. m. malerkotliana } & $\mathrm{RC}$ & $1(0-1)$ & $3(2-4)$ & $1(1-2)$ & $2(1-3)$ & $6.49 \pm 0.12(3-9)$ \\
\hline & $\mathrm{BP}$ & $1(0-2)$ & $3(1-4)$ & $1(1-2)$ & $2(1-3)$ & $6.32 \pm 0.11(4-10)$ \\
\hline & MY & $1(0-2)$ & $3(2-4)$ & $1(1-2)$ & $2(1-3)$ & $7.33 \pm 0.12(5-10)$ \\
\hline & BHU & $1(0-1)$ & $2(1-4)$ & $1(0-1)$ & $2(1-3)$ & $5.29 \pm 0.12(3-10)$ \\
\hline & & & Mean & & & $6.35 \pm 0.06$ \\
\hline \multirow[t]{2}{*}{ D. p. nigrens } & $\mathrm{CM}$ & $2(1-3)$ & $3(3-4)$ & $2(1-4)$ & 0 & $7.88 \pm 0.13(4-10)$ \\
\hline & & & & & Mean & $7.88 \pm 0.13$ \\
\hline
\end{tabular}

the PN strain no such variation was found. There is also variation in the number of teeth in the two sets of the distal comb: for the TD strain, 1/6, 1/7,1/9, 2/8, 2/9, 3/5, $3 / 6,3 / 7,3 / 9,4 / 5,4 / 6,4 / 7,5 / 1$ (upper set /lower set) and in only one male three sets were observed, where the number of teeth is $1 / 2 / 8$ (upper set /lower set /lowest set); for the AR strain, $1 / 7$ and $1 / 10$; for the NG strain, 1/7, $1 / 8,1 / 9$ and $2 / 9$; for the $\mathrm{AD}$ strain, $1 / 7$ and $2 / 4$; for the PN strain, $1 / 7$; for the SL strain $1 / 5$ and $5 / 2$; and for the MY strain, no such variation was observed. In the second tarsomere, there is much less variation in teeth number in comparison to the first tarsomere and only in some males of TD, NG and PN strains, two sets were observed instead of a single sex comb set. In the TD strain, the variation in the number of teeth on the second tarsomere is $1 / 1,1 / 2,1 / 3$ (upper set / lower set) while in the NG and PN strains, they are $1 / 1,1 / 2$ (upper set / lower set) and 1/1(upper set / lower set), respectively.

D. parabipectinata exhibits minimum variation in sex comb patterns (Figs 2a-b, Table 2B). There are two sets instead of a single set in the proximal comb of first tarsomere in some males as in the case of $D$. b. bipectinata.

TABLE 2B. Intraspecific variability in sex comb teeth number in different species of the D. bipectinata complex.

\begin{tabular}{|c|c|c|c|c|c|}
\hline Species & Strains & Mean \pm SD & $\mathrm{CV}$ & $\chi^{2 \#}$ & P-value \\
\hline \multirow{7}{*}{ D. $b$. bipectinata } & TD & $15.93 \pm 1.506$ & 0.095 & \multirow{7}{*}{18.2} & \multirow{7}{*}{$<0.01 * *$} \\
\hline & $\mathrm{AR}$ & $15.69 \pm 1.433$ & 0.091 & & \\
\hline & MY & $14.24 \pm 1.372$ & 0.096 & & \\
\hline & NG & $15.73 \pm 1.510$ & 0.096 & & \\
\hline & $\mathrm{AD}$ & $13.54 \pm 1.459$ & 0.108 & & \\
\hline & $\mathrm{PN}$ & $14.02 \pm 1.670$ & 0.119 & & \\
\hline & SL & $14.19 \pm 1.760$ & 0.119 & & \\
\hline \multirow{2}{*}{ D. parabipectinata } & $\mathrm{CL}$ & $15.06 \pm 1.613$ & 0.107 & \multirow{2}{*}{5.198} & \multirow{2}{*}{$<0.05^{*}$} \\
\hline & MY & $14.16 \pm 1.916$ & 0.135 & & \\
\hline \multirow{4}{*}{ D. m. malerkotliana } & $\mathrm{BP}$ & $6.32 \pm 1.081$ & 0.171 & \multirow{4}{*}{13.74} & \multirow{4}{*}{$<0.01 * *$} \\
\hline & BHU & $5.29 \pm 1.192$ & 0.225 & & \\
\hline & MY & $7.33 \pm 1.164$ & 0.159 & & \\
\hline & $\mathrm{RC}$ & $6.48 \pm 1.590$ & 0.179 & & \\
\hline D. p. nigrens & $\mathrm{CM}$ & $7.88 \pm 1.372$ & 0.174 & & \\
\hline
\end{tabular}

${ }^{\#}$ Evaluating test for homogeneity for intraspecific co-efficient of variation $(\mathrm{CV}){ }^{*},{ }^{* *}$ Significant . 


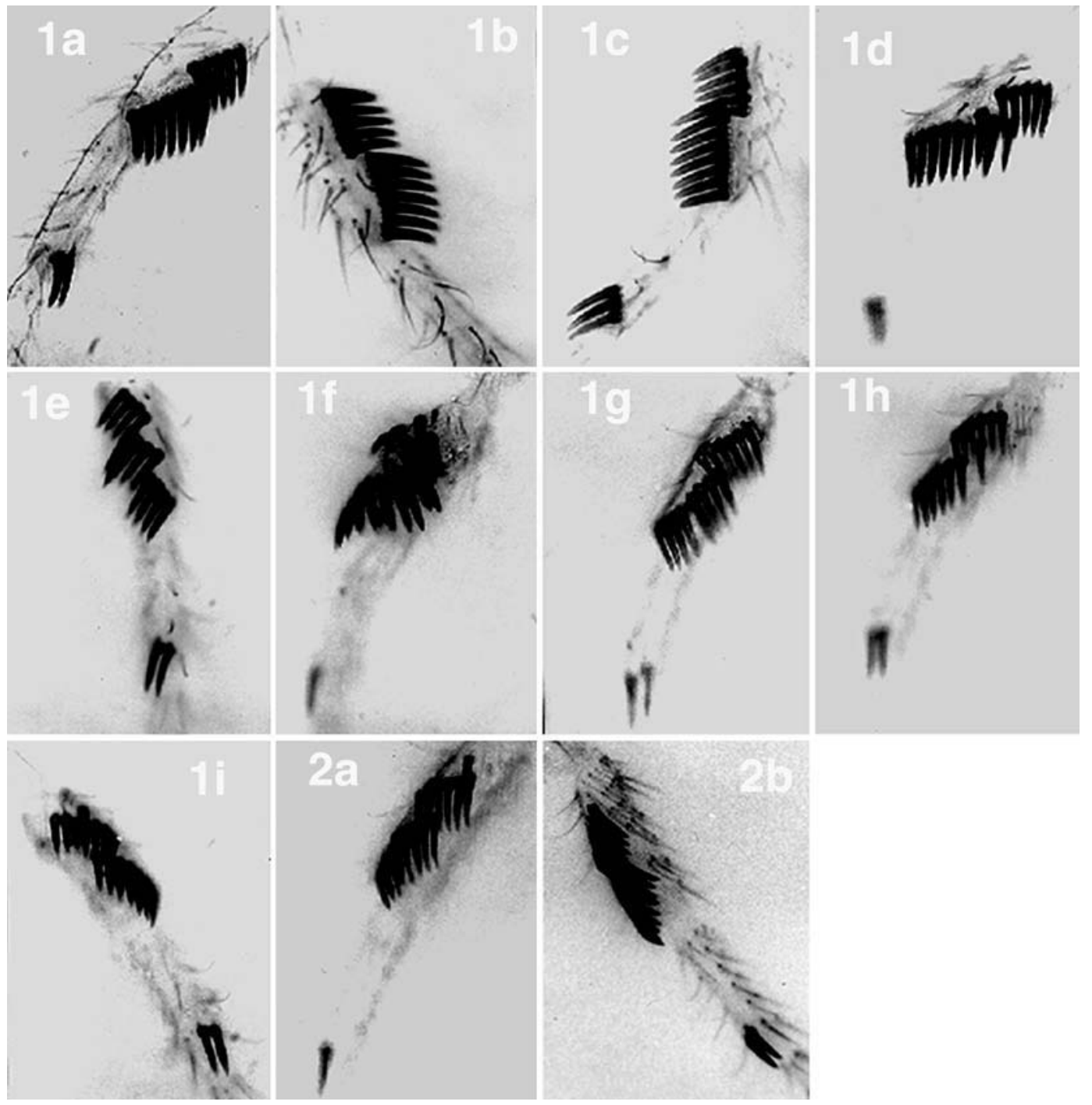

Figs 1-2: Sex comb patterns. $1 \mathrm{a}-\mathrm{i}-$ D. b. bipectinata; $2 \mathrm{a}-\mathrm{b}-$ D. parabipectinata.

For example, in the CL strain, the number of teeth is $1 / 4$, $1 / 5,1 / 6,1 / 7,2 / 5,2 / 6,2 / 7,3 / 7$ and $6 / 2$ (upper set / lower set), while in the MY strain, it is $1 / 3,1 / 4,1 / 5,4 / 2,5 / 1$ and $5 / 2$ (upper set / lower set). However, the variation in teeth number on the second tarsomere is low. Here also, two sets are found instead of a single set in the distal comb of the first tarsomere where only one variant is observed both in the CL (6/1 - upper set / lower set) and the MY (1/7 - upper set / lower set) strains. No such variation was observed for the second tarsomere of both strains. The sex combs on first tarsomere in both species (bipectinata and parabipectinata) are arranged in longitudinal rows (Bock, 1971a).

Among the four investigated species, D. malerkotliana is unique in exhibiting four sex combs: two combs on both the first and second tarsomeres. The total teeth number of $D$. m. malerkotliana is lower (almost half) than that of $D$. b. bipectinata and $D$. parabipectinata (Table 2A). It exhibits higher variation in sex comb patterns (Figs 3a-f) than D. parabipectinata (Figs 2a-b, Table 2B) and $D$. p. nigrens (Figs $4 \mathrm{a}-\mathrm{c}$ ). As in D. b. bipectinata and D. parabipectinata, D. m. malerkotliana also has two sets instead of a single set in the proximal comb of the second tarsomere in the MY strain, having teeth number $1 / 1$ and $1 / 2$ (upper set / lower set), but no such variation is recorded in other combs. In other strains, no such variation is observed in any comb. The total number of teeth in $D$. $p$. nigrens is higher than that of $D$. m. malerkotliana but lower than that of $D$. b. bipectinata and D. parabipectinata (Table 2A). However, the variation in sex comb phenotypes in this taxon (Figs $4 \mathrm{a}-\mathrm{c}$ ) is higher than that of $D$. parabipectinata but lower than that of $D$. b. bipectinata and D. m. malerkotliana. There was no record of two sets instead of a single sex comb set in D. p. nigrens. However, their presence cannot be ruled out in other strains of D. p. nigrens. Sex combs on the first tarsomere are arranged transversely both in $D$. m. malerkotliana and D. p. nigrens (Bock, 1971a).

The four species have been compared for variability in teeth number within the strain and also between the strains that revealed higher variability in $D$. b. bipectinata and $D . m$. malerkotliana than $D$. parabipectinata (Table 2B). Intraspecific variation in teeth number of seven $D$. b. bipectinata strains and four $D . m$. malerkot- 

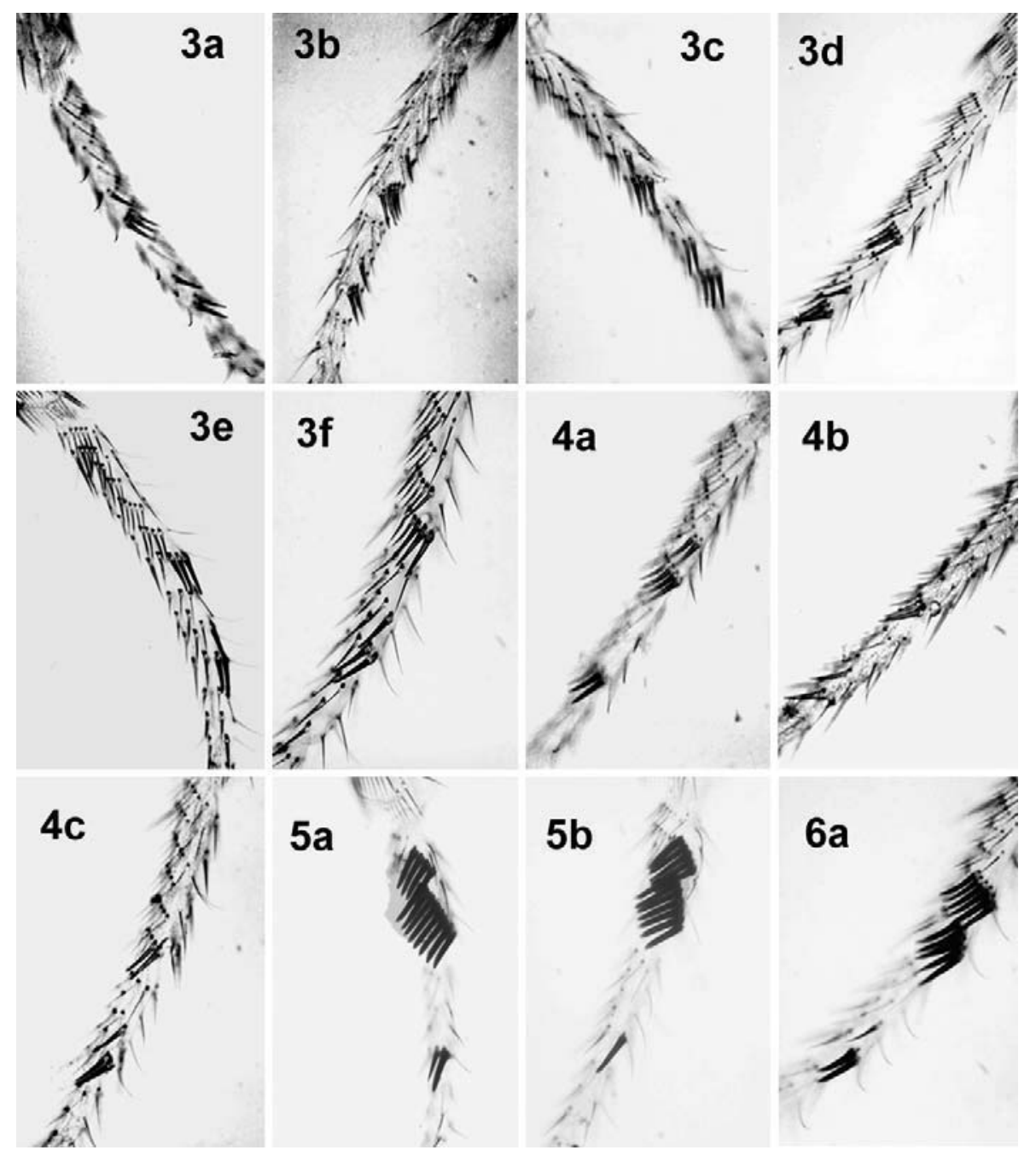

Figs 3-6a: Sex comb patterns. 3a-f - D. m. malerkotliana; 4a-c - D. p. nigrens; 5a-b - hybrid of female D. b. bipectinata $\times$ male D. parabipectinata; $6 \mathrm{a}-$ hybrid of female $D$. b. bipectinata $\times$ male D. m. malerkotliana.

liana strains shows highly significant differences (Tables $3 \mathrm{~A}$ and $\mathrm{B}$ ). A t-test of intraspecific difference of mean number of teeth between two strains of $D$. parabipectinata shows a highly significant difference $(\mathrm{t}=3.7, \mathrm{df}=$ $198, p<0.001)$. Further, the interspecific variation in mean number of teeth among the four species using one strain (the same strain which was used for hybridization experiment) of each species has been calculated by employing ANOVA. Interestingly, here also we get a statistically highly significant difference (Table $3 \mathrm{C}$ ). The pairwise comparisons among the four species using the same strains as above have been performed using DMRT statistical tool and, as expected, there is significant differ-

TABLE 3. Analysis of variance (ANOVA) for interspecific and intraspecific variation in the mean number of sex comb teeth.

\begin{tabular}{|c|c|c|c|c|c|c|}
\hline & Source & df & SS & MS & $\mathrm{F}$ & $\mathrm{P}$ \\
\hline & Total & 699 & 2214.63 & & & \\
\hline \multirow[t]{3}{*}{ A D. b. bipectinata strains } & Between strains & 6 & 580.60 & 96.77 & 41.04 & $<0.001 *$ \\
\hline & Within strains & 693 & 1634.00 & 2.36 & & \\
\hline & Total & 399 & 733.88 & & & \\
\hline \multirow[t]{3}{*}{ B D. m. malerkotliana strains } & Between strains & 3 & 210.43 & 70.14 & 53.06 & $<0.001 *$ \\
\hline & Within strains & 396 & 523.45 & 1.32 & & \\
\hline & Total & 399 & 6259.39 & & & \\
\hline \multirow[t]{2}{*}{ C D. bipectinata complex } & Between strains & 3 & 5866.11 & 1955.37 & 1968.87 & $<0.001^{*}$ \\
\hline & Within strains & 396 & 393.29 & 0.99 & & \\
\hline
\end{tabular}

* Significant. 


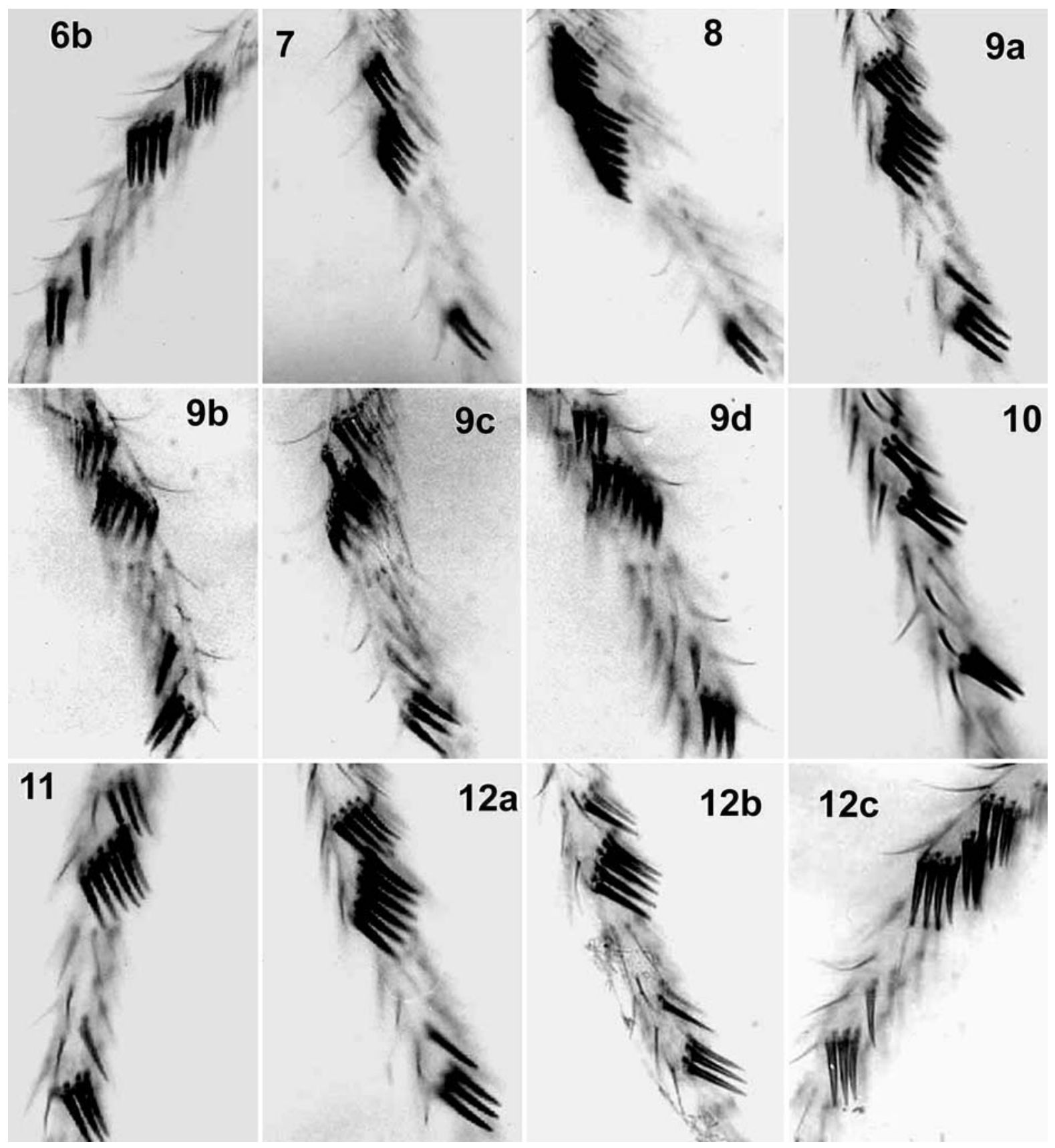

Figs 6b-12: Sex comb patterns. $6 \mathrm{~b}-$ hybrid of female D. b. bipectinata $\times$ male D. m. malerkotliana; 7 - hybrid of female $D$. $b$. bipectinata $\times$ male $D$. p. nigrens; 8 - hybrid of female $D$. parabipectinata $\times$ male $D$. b. bipectinata; 9 a- $\mathrm{d}-$ hybrid of female $D$. parabipectinata $\times$ male D. m. malerkotliana; 10 - hybrid of female D. parabipectinata $\times$ male D. p. nigrens; 11 - hybrid of female D. m. malerkotliana $\times$ male $D$. b. bipectinata $; 2 \mathrm{a}-\mathrm{c}-$ hybrid of female $D$. m. malerkotliana $\times$ male $D$. parabipectinata .

ence in all species except between $D$. b. bipectinata and D. parabipectinata (Table 4).

The degree of hybridization among the four species varied. It is easier to obtain hybrids between $D$. $b$. bipectinata and D. parabipectinata and when D. b. bipectinata was the female parent, the degree of hybridization was comparatively higher. When these two species are crossed with $D$. m. malerkotliana, the hybridization is a bit more difficult. Finally, in the crosses where D. $p$. nigrens is one of the parental species, obtaining hybrids is difficult (for degree of crossability, see Mishra \& Singh, 2006).
The range and mean teeth number and number of teeth in each comb of both tarsomeres for the hybrids are given in Table 5, and their variations are shown in Figs 5a-16g. The pattern of sex comb teeth in hybrids is intermediate of their parental species. In some hybrids, there are two sets instead of a single comb set on the first and second tarsomeres. However, their frequency is less than $10 \%$. For example, in the hybrids between $D$. parabipectinata females and $D$. b. bipectinata males, instead of a single set of proximal comb, there are two sets with teeth number $1 / 5$ (upper set /lower set). Similarly, instead of a single distal comb set, there are two sets with teeth 


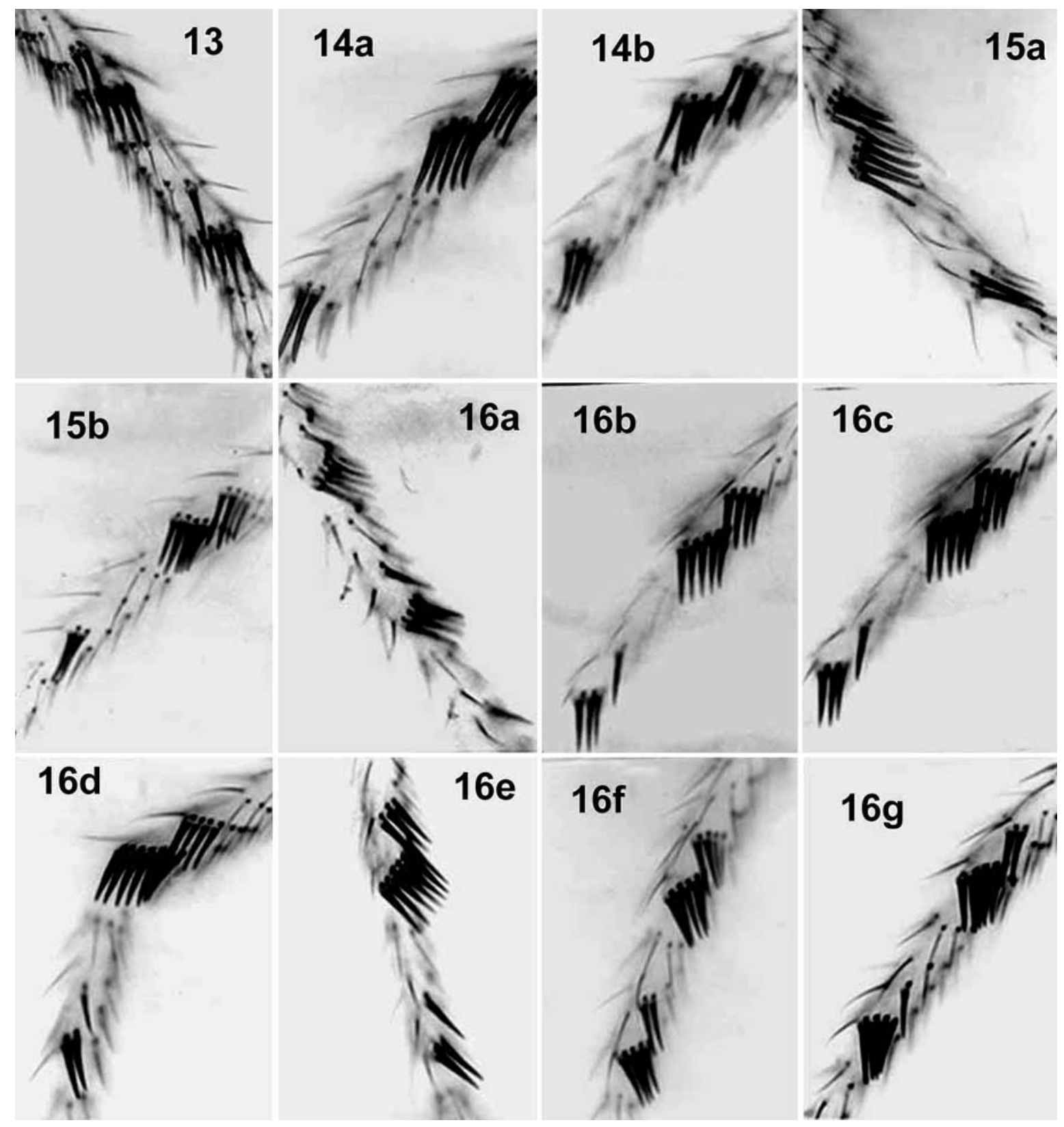

Figs 13-16: Sex comb patterns. 13 - hybrid of female $D$. m. malerkotliana $\times$ male $D$. p. nigrens; $14 \mathrm{a}-\mathrm{b}-$ hybrid of female $D$. $p$. nigrens $\times$ male $D . b$. bipectinata; $15 \mathrm{a}-\mathrm{b}-$ hybrid of female $D$. p . nigrens $\times$ male $D$. parabipectinata; $16 \mathrm{a}-\mathrm{g}-$ hybrid of female $D$. $p$. nigrens $\times$ male $D$. m. malerkotliana.

number $7 / 1$ (upper set /lower set). The same pattern is found in the hybrids between male $D$. b. bipectinata and female $D$. p. nigrens, where two sets having teeth numbers $1 / 3$ and $1 / 5$ (upper set /lower set) are present instead of a single proximal comb set, and $1 / 5$ and $2 / 5$ teeth are present instead of a single distal comb set. The hybrids between $D$. parabipectinata and D. m. malerkotliana have four sex combs, two combs both on the first and second tarsomeres. In this case, the variability in comb pattern is higher when $D$. parabipectinata is the female parent. The number of teeth in the two proximal comb sets on first tarsomere is $1 / 4,2 / 1,3 / 2$ (upper set /lower set) and in the distal comb is $6 / 1,7 / 1$ (upper set /lower set). Further, in the proximal comb of second tarsomere, there is only one variant where the two sets instead of a single set have $1 / 1$ (upper set /lower set) teeth. In the reciprocal cross (female parent is D. m. malerkotliana), there is only one variant for each comb on both tarsomeres; for proximal and distal combs of first tarsomere, it is $3 / 2$ and $3 / 4$ (upper set /lower set), respectively, while for proximal and distal combs of second tarsomere, it is $1 / 1$ and $2 / 1$ (upper set /lower set), respectively. In the hybrids between female $D$. m. malerkotliana and male $D$. p. nigrens, only one variant is present where the single distal comb set of the second tarsomere is replaced with two sets having teeth number 2/1 (upper set /lower set). Similarly, in the hybrids between female $D$. p. nigrens and male D. parabipectinata, instead of a single distal 
TABLE 4. DMRT for pairwise comparison among four species of the D. bipectinata complex.

\begin{tabular}{lccc}
\hline Comparisons & Difference of means & Critical difference & $P$ \\
\hline bip vs parab & 0.10 & 2 & $>0.05$ \\
bip vs maler & 8.40 & 4 & $<0.05^{*}$ \\
parab vs maler & 8.30 & 3 & $<0.05^{*}$ \\
bip vs pseu & 6.87 & 3 & $<0.05^{*}$ \\
parab vs pseu & 6.77 & 2 & $<0.05^{*}$ \\
maler vs pseu & 1.53 & 2 & $<0.05^{*}$
\end{tabular}

bip $=D$. b. bipectinata $;$ parab $=D$. parabipectinata $;$ maler $=D$. m. malerkotliana $;$ sseu $=$ D. p. nigrens $;{ }^{*}$ Significant.

comb set on the second tarsomere, there are two sets with teeth number $1 / 1$ and $1 / 2$ (upper set /lower set).

Although, in most crosses, the teeth number in hybrids differs from their midparent value (average of teeth number of parental species involved in a cross), they are always intermediate of their parental species (Table 6). Further, the teeth number of hybrids differs from maternal and paternal species in all crosses except few crosses where they are similar with either the maternal or the paternal parents (Table 6). Reciprocal crosses show similar teeth number with only two exceptions (Table 6).

\section{DISCUSSION AND CONCLUSION}

$D$. b. bipectinata and $D$. parabipectinata have a similar sex comb pattern (Table 2), so it is very difficult to distinguish the two species based on this character. However, they can be easily distinguished by the sharp contrast in the abdominal tip pigmentation between males of the two species: D. bipectinata has a pale brown abdominal tip while D. parabipectinata has a melanized abdominal tip. Nevertheless, D. b. bipectinata and D. parabipectinata can be easily distinguished from $D$. m. malerkotliana and D. p. nigrens on the basis of sex comb patterns. As in $D$. $b$. bipectinata and $D$. parabipectinata, the number of teeth in $D$. p. nigrens is lesser in the proximal comb than in the distal comb of the first tarsomere and the number of teeth on the second tarsomere is similar to that on the proximal comb of the first tarsomere (Table 2). In the above three species there are only three combs: two combs on the first tarsomere and one comb on the second, but in D. malerkotliana there are four combs: two combs on both the first and the second tarsomeres. Further, the number of teeth is lesser in the proximal than in the distal combs on both tarsomeres (Table 2). The sex comb teeth of both D. bipectinata and D. parabipectinata are thicker and more prominent than in D. pseudoananassae and $D$. malerkotliana, where teeth are slightly tougher and thicker than the bristles (Figs 1a-4c). Species similarities in sex comb patterns are consistent with the phylogenetic relationship derived from cytological (Bock, 1971b), biochemical (Yang et al., 1972; Hegde \& Krishnamurthy, 1976), morphological (Mishra \& Singh, 2006) and molecular studies (Kopp \& Barmina, 2005). Further, the sex comb patterns of these four species is well in line with the hypothesis that "as far as phylogeny may be reconstructed, malerkotliana, pseudoananassae and a population ancestral to bipectinata and parabipectinata are derived directly from a common ancestral population" (Bock, 1971b). Therefore, similarity in sex comb patterns may be correlated with the phylogenetic proximity of these species.

From the results, it is obvious that the variation in sex comb patterns is highest in D. b. bipectinata (Figs 1a-i). The interstrain variability in teeth number is also higher (Table 2B), and some unique sex comb phenotypes are also observed in this species. Further, D. b. bipectinata also shows a tendency to expend its geographical distribution (Yang et al., 1972). Notably, there is more variation in teeth patterns in the proximal comb of the first tarsomere in $D$. b. bipectinata. This might be the result of higher sexual selection of these teeth during evolution (Polak et al., 2004). In D. parabipectinata, although the sex comb pattern is similar to that of $D$. bipectinata, it has

TABLE 5. Details of sex comb teeth arrangements in interspecific hybrids of the D. bipectinata species complex.

\begin{tabular}{|c|c|c|c|c|c|c|}
\hline \multirow[b]{2}{*}{ Crosses } & \multirow{2}{*}{$\begin{array}{l}\text { Range of total } \\
\text { teeth no. }\end{array}$} & \multirow{2}{*}{$\begin{array}{l}\text { Mean } \\
\text { teeth no. }\end{array}$} & \multicolumn{2}{|c|}{ First tarsomere } & \multicolumn{2}{|c|}{ Second tarsomere } \\
\hline & & & $\begin{array}{l}\text { Proximal comb } \\
\text { teeth no. (range) }\end{array}$ & $\begin{array}{c}\text { Distal comb } \\
\text { teeth no. (range) }\end{array}$ & $\begin{array}{l}\text { Proximal comb } \\
\text { teeth no. (range) }\end{array}$ & $\begin{array}{c}\text { Distal comb } \\
\text { teeth no. (range) }\end{array}$ \\
\hline bip $\times$ parab & $12-16$ & $14.38 \pm 0.11$ & $5(4-7)$ & $8(5-9)$ & $2(1-3)$ & 0 \\
\hline parab $\times$ bip & $12-16$ & $14.47 \pm 0.10$ & $5(4-7)$ & $7(6-9)$ & $2(1-2)$ & 0 \\
\hline bip $\times$ maler & $10-15$ & $12.94 \pm 0.14$ & $4(2-6)$ & $5(4-7)$ & $1(0-1)$ & 3 \\
\hline maler $\times$ bip & $10-14$ & $11.77 \pm 0.10$ & $4(2-5)$ & $5(4-7)$ & $1(0-2)$ & 2 \\
\hline bip $\times$ pseu & $9-14$ & $11.98 \pm 0.15$ & $4(2-5)$ & $6(5-7)$ & $3(1-4)$ & 0 \\
\hline pseu $\times$ bip & $9-14$ & $11.91 \pm 0.14$ & $4(1-6)$ & $6(4-7)$ & $2(1-4)$ & 0 \\
\hline parab $\times$ maler & $10-15$ & $12.86 \pm 0.13$ & $3(2-4)$ & $6(4-7)$ & $1(0-2)$ & $2(2-4)$ \\
\hline maler $\times$ parab & $9-15$ & $12.13 \pm 0.12$ & $3(2-4)$ & $5(4-7)$ & $1(0-2)$ & $2(2-3)$ \\
\hline parab $\times$ pseu & $8-13$ & $11.03 \pm 0.13$ & $4(2-6)$ & $6(3-7)$ & $2(1-4)$ & 0 \\
\hline pseu $\times$ parab & $8-13$ & $11.11 \pm 0.13$ & $4(1-5)$ & $6(3-7)$ & $2(1-3)$ & 0 \\
\hline maler $\times$ pseu & $5-9$ & $7.56 \pm 0.09$ & $0(0-1)$ & $4(3-5)$ & $1(1-2)$ & $2(2-3)$ \\
\hline pseu $\times$ maler & $6-9$ & $7.71 \pm 0.09$ & $1(0-2)$ & $3(3-4)$ & $1(0-1)$ & $3(2-4)$ \\
\hline
\end{tabular}

bip $=D$. b. bipectinata $;$ parab $=D$. parabipectinata $;$ maler $=D$. m. malerkotliana $;$ pseu $=D . p$. nigrens. 
TABLE 6. Mean number of sex comb teeth in the interspecific hybrids and their comparison with parental species and midparent value (comparison between reciprocal crosses is also given).

\begin{tabular}{lcccccc}
\hline Female $\times$ Male & Mean \pm SE & Midparent value & $\mathrm{t}_{1}$ & $\mathrm{t}_{2}$ & $\mathrm{t}_{3}$ & $\mathrm{t}_{4}$ \\
\hline bip $\times$ parab & $14.38 \pm 0.11$ & 14.10 & 1.61 & 1.81 & 0.88 & 0.60 \\
parab $\times$ bip & $14.47 \pm 0.10$ & & $2.19^{*}$ & 1.31 & $2.30^{*}$ & \\
bip $\times$ maler & $12.94 \pm 0.14$ & 10.35 & $13.74^{* * *}$ & $5.01^{* * *}$ & $36.07^{* * *}$ & $6.87^{* * *}$ \\
maler $\times$ bip & $11.77 \pm 0.10$ & & $8.35^{* * *}$ & $34.24^{* * *}$ & $11.49^{* * *}$ & \\
bip $\times$ pseu & $11.98 \pm 0.15$ & 10.94 & $5.65^{* * *}$ & $8.99^{* * *}$ & $20.28^{* * *}$ & 0.34 \\
pseu $\times$ bip & $11.91 \pm 0.14$ & & $5.67^{* * *}$ & $21.17^{* * *}$ & $9.74^{* * *}$ & \\
parab $\times$ maler & $12.86 \pm 0.13$ & 10.34 & $14.24^{* * *}$ & $5.85^{* * *}$ & $36.77^{* * *}$ & $4.12^{* * *}$ \\
maler $\times$ parab & $12.12 \pm 0.12$ & & $10.22^{* * *}$ & $33.03^{* * *}$ & $9.19^{* * *}$ & \\
pseu $\times$ parab & $11.11 \pm 0.13$ & 11.03 & 0.49 & $13.45^{* * *}$ & $17.37^{* * *}$ & 0.43 \\
parab $\times$ pseu & $11.03 \pm 0.13$ & & 0.03 & $16.96^{* * *}$ & $13.81^{* * *}$ & 1.96 \\
maler $\times$ pseu & $7.56 \pm 0.09$ & 7.18 & $2.81 * *$ & $7.09^{* * *}$ & 2.06 & 1.02 \\
pseu $\times$ maler & $7.71 \pm 0.09$ & & $3.84^{* * *}$ & 1.09 & $7.97^{* * *}$ &
\end{tabular}

bip $=D . b$. bipectinata $;$ parab $=$ D. parabipectinata $;$ maler $=D . m$. malerkotliana $;$ pseu $=D . p$. nigrens. Midparent value $=$ average of teeth number of parental species involved in a cross. $t_{1}$, difference between hybrid and midparent value; $t_{2}$, difference between hybrid and maternal species; $\mathrm{t}_{3}$, difference between hybrid and paternal species and $\mathrm{t}_{4}$, difference between reciprocal hybrids. ${ }^{*} \mathrm{p}<$ $0.05 ; * * \mathrm{p}<0.01 ; * * * \mathrm{p}<0.001$.

less variation in sex comb phenotypes. It also has a restricted geographical distribution. The diversity of teeth patterns in D. m. malerkotliana is greater than in $D . p$. nigrens and its geographical distribution is also wider. These findings elicit that species having wider biogeographical distributions endow with higher diversity, while species with restricted distributions have comparatively less diversity of sex comb patterns.

The degree of hybridization among the four species indicates their genetic compatibility, which in turn reflects their phylogenetic proximity. The degree of crossability is higher between $D$. bipectinata and $D$. parabipectinata than that of D. bipectinata or D. parabipectinata and D. malerkotliana, and it is lowest between D. malerkotliana and D. pseudoananassae (Mishra \& Singh, 2006). These observations reinforce that $D$. bipectinata and $D$. parabipectinata are very closely related species. These species are closer to D. malerkotliana but distantly related with $D$. pseudoananassae.

In the hybrids of these four species, the number of teeth is intermediate of their parental species. However, when the difference of teeth number of the hybrids and the midparent value of their corresponding parental species are statistically tested, no fixed pattern is found. In some hybrids $(D . b$. bipectinata $\times D . m$. malerkotliana, $D$. parabipectinata $\times D . m$ malerkotliana, D. b. bipectinata $\times D$. $p$. nigrens $)$, the difference is highly significant $(\mathrm{p}<$ 0.001 ), while in others it is less significant as observed in the hybrids of female $D$. m. malerkotliana and male $D . p$. nigrens $(\mathrm{p}<0.01)$ and female $D$. parabipectinata and male D. b. bipectinata $(\mathrm{p}<0.05)$, or not significant $(\mathrm{p}>$ 0.05 ) as in the case of hybrids of female $D$. $b$. bipectinata and male $D$. parabipectinata, and reciprocal crosses of $D$. $p$. nigrens and $D$. parabipectinata (Table 6). These observations support a polygenic mode of inheritance of sex combs. However, our results slightly differ from Crossley \& Taylor (1985) who, although reported intermediate sex comb patterns in hybrids, found no significant difference between teeth number of the hybrids and their corresponding midparent values. The reason for this difference may be attributed to the following factors: (1) Quantitative Trait Loci (genetic factors underlying quantitative traits) at different regions are responsible for higher and lower number of sex comb teeth (Nuzhdin \& Reitwich, 2000). The expression of higher QTL number may increase the teeth number in hybrids compared to their midparent value and (2) the mutations in genes in the bristle formation pathway could perhaps also modify teeth number (Mackay, 1995). Further, there is a significant difference in teeth number in different strains of the same species, and even among different individuals of the same strain (Table 2). These findings corroborate the genetic heterogeneity in teeth number and its additive effect.

Sex comb in males of Drosophila (Sophophora) is a diversifying secondary sexual character, which is sexually selected for size and symmetry (Polak et al., 2004). In most Sophophora species, there are two sex combs, one on the first tarsomere and another on the second. However, variation in comb patterns is found in different species of the subgenus Sophophora. D. melanogaster, the most extensively studied species, has only one sex comb (Santamaria, 1998). However, Drosophila species other than those in the subgenus Sophophora entirely lack sex combs as it is the case of the saltans and willistoni groups. Considering these variability, Stern (1954) speculated that the evolutionary process which diversified sex comb phenotypes in different species began in response to mutation to preexisting developmental prepatterns. An interesting query regarding the genetic basis of inheritability of teeth number is whether the same gene is responsible for both intra- and interspecific variability of this trait. Nuzhdin \& Reiwitch (2000) tried to unravel this mystery. They found that the same chromosome region has contributed both to intraspecific variability in $D$. 
melanogaster and interspecific difference between $D$. mauritiana and D. simulans.

The functional significance of sex comb is also a matter of discussion. Different workers empirically assigned different functions to sex combs, all functions pertaining to the sexual behaviour of males. Spieth (1952) found a distinct correlation between the behaviour used by males during mating and the presence of sex combs. Males with sex combs attempt intromission before mounting is complete, but males without sex combs do not. Based on this, he speculated that sex combs are necessary in precopulatory maneuvering by males. Narda (1968) observed elimination of tapping by removing first and second tarsomeres that decreases considerably the sexual isolation between $D$. bipectinata and D. malerkotliana. It is also speculated that sex combs are scent receptors and teeth number reflects evolutionary divergence in response to species-specific scents (Crossley \& Taylor, 1985). In $D$. silvestris, it has been demonstrated that cilia on the first and the second tarsomeres of male foretarsi are used as a brush to stimulate the female during courtship (Carson \& Tremoto, 1984). Sex combs are used during both courtship and copulation in D. melanogaster and the copulatory success of males decreases when sex combs are surgically removed (Speith, 1952; Coyne, 1985). Males lacking the foreleg tarsomere with the sex comb have great difficulty in grasping the female genitalia resulting into substantial decrease in mating ability (Cook, 1977; Coyne, 1985). Moreover, the role of sex combs as precise grasping organ is reinforced by the presence of small cuticular projections at the base of each tooth (Coyne, 1985) that may provide mechanosensory information to the males. A row of bristles was found near the tip of the female ovipositor (Coyne, 1985), which may act as an anchor to the male sex comb bristles during copulation. Therefore, it can be suggested that sex combs are primarily structures adapted for grasping the female securely during the act of intromission. Further, it has been documented that the number of teeth and their positioning are perhaps under sexual selection that causes rapid changes in sex comb morphology and correlated changes in mating behaviour (Carson \& Lander, 1984). Obeying this selection process, sex combs strongly differ in the number of rows, and in their position and orientation among races and species. These variations are manifested in D. bipectinata (Figs 1b-i).

In summary, (1) sex comb patterns are correlated with the phylogenetic relationship in closely related species, at least in the four members of the D. bipectinata complex, (2) the degree of hybridization is higher in phylogenetically closely related species and lower in distantly related species, (3) species with wider geographical distribution have higher divergence in sex comb phenotypes than those with restricted geographical distribution, (4) interspecific hybrids show intermediate sex comb patterns and no fixed relationship was found between the midparent values and sex comb teeth number of hybrids, which corroborates a polygenic mode of inheritance, and (5) it is documented that sex combs are a secondary sexual trait primarily used by males for grasping females during intromission.

Understanding the genetic basis of inheritance of sex combs and their divergence during evolution is only the first step to catch the historical context of the evolution of this trait. To understand the pattern of this trait in ancestral population and the sequence of genetic changes involved during change of its phenotypes, further studies are required.

ACKNOWLEDGEMENTS. Financial support in the form of Senior Research Fellowship (SRF) of Centre of Advanced Study, Department of Zoology, Banaras Hindu University to P.K. Mishra is gratefully acknowledged. We thank I.R. Bock for providing $D$. parabipectinata CL stock, P. Štys (editor) and two anonymous reviewers for their helpful comments on the original draft of the manuscript.

\section{REFERENCES}

Bock I.R. 1971a: Taxonomy of the Drosophila bipectinata species complex. Univ. Texas Publ. 7103: 273-280.

Bock I.R. 1971b: Intra- and interspecific chromosomal inversions in the Drosophila bipectinata species complex. Chromosoma 34: 206-229.

Bock I.R. 1978: The bipectinata complex: A study in interspecific hybridization in the genus Drosophila (Insecta; Diptera). Aust. J. Biol. Sci. 31: 197-208.

Bock I.R. \& WheELer M.R. 1972: The Drosophila melanogaster species group. Univ. Texas Publ. 7213: 1-102.

CARSON H.L. \& LANDER R. 1984: Inheritance of a secondary sexual character in Drosophila silvestris. Proc. Nat. Acad. Sci. USA 81: 6904-6907.

Carson H.L. \& Teramoto L.T. 1984: Artificial selection for a secondary sexual character in males of Drosophila silvestris from Hawaii. Proc. Nat. Acad. Sci. USA 81: 3915-3917.

Cook R.M. 1977: Behavioral role of sex combs in Drosophila melanogaster and Drosophila simulans. Behav. Genet. 7: 349-357.

CoYne J.A. 1985: Genetic studies of three sibling species of Drosophila with relationship to theories of speciation. Genet. Res. 46: 169-192.

Crossley S.A. \& TAYlor I. 1985: Male tarsal sex comb teeth pattern in the Drosophila bipectinata complex. Experientia 41: 103-104.

Gupta J.P., Dwivedi Y.N. \& Singh B.K. 1980: Natural hybridization in Drosophila. Experientia 36: 290.

Hegde S.N. \& Krishnamurthy N.B. 1976: Studies on the genetics of isozymes in the hybrids of Drosophila bipectinata complex. Aust. J. Zool. 27: 421-431.

Kopp A. \& BARMina O. 2005: Evolutionary history of the Drosophila bipectinata species complex. Genet. Res. 85: 23-46.

Lachaise D. \& Chassagnard M. 2002: Divergence of sex comb phenotypes in the Drosophila fima species group and radiation on Afrotropical Ficus, including five new species from East Africa and Madagascar (Diptera: Drosophilidae). Ann. Soc. Entomol. Fr. (N.S.) 38: 79-99.

MACKAY T.F.C. 1995: The genetic basis of quantative variation: number of sensory bristles of Drosophila melanogaster as a model system. Trends Genet. 11: 464-470.

Matsuda M., Tomimura Y. \& Tobari Y.N. 2005: Reproductive isolation among biogeographical populations of Drosophila bipectinata Duda (Diptera, Drosophilidae) with recognition of three subspecies. Genetica 125: 69-78. 
Mishra P.K. \& Singh B.N. 2005a: Genetic basis of hybrid male sterility among three closely related species of Drosophila. Ind. J. Expt. Biol. 43: 455-461.

Mishra P.K. \& Singh B.N. 2005b: Why hybrid males are sterile in Drosophila? Curr. Sci. 11: 1813-1819.

Mishra P.K. \& Singh B.N. 2006: Drosophila bipectinata species complex: Study of phylogenetic relationship among four members through the analysis of morphology of testes and seminal vesicles. J. Zool. Syst. Evol. Res. 44: 175-179.

NARDA R.D. 1968: Experimental evaluation of the stimuli involved in sexual isolation among three members of the ananassae species subgroup (Sophophora, Drosophila). Anim. Behav. 16: 117-119.

Nuzhdin S.V. \& Reiwitch S.G. 2000: Are the same genes responsible for intra- and interspecific variability for sex comb tooth number in Drosophila? Heredity 84: 97-102.

OKADA T. 1981: Oriental species, including New Guinea. In Ashburner M., Carson H.L. \& Thompson J.N. Jr. (eds): Genetics of Drosophila. Vol. 3a. Academic Press, London, pp. 261-289.

Polak M., Starmer W.T. \& Wolf L.L. 2004: Sexual selection for size and symmetry in a diversifying secondary sexual character in Drosophila bipectinata Duda (Diptera: Drosophilidae). Evolution 58: 597-607.

SANTAMaria P. 1998: Genesis versus epigenesis: the odd jobs of the polycomb group of genes. Int. J. Dev. Biol. 42: 463-469.

SINGH S. \& SingH B.N. 2001: Drosophila bipectinata species complex. Ind. J. Exp. Biol. 39: 835-844.

SPIETH H.T. 1952: Mating behaviour within the genus Drosophila (Diptera). Bull. Am. Mus. Nat. Hist. 99: 395-474.

Stern C. 1954: Genes and developmental patterns. Proc. IX Intern. Congr. Genet. Part I. Caryologia (Pisa) 6 (Suppl.): 355-369.

Tomimura Y., Matsuda M. \& Tobari Y.N. 2005: Chromosomal phylogeny and biogeographical divergence in the Drosophila bipectinata complex. Genome 48: 487-502.

YANG S.Y., WHEELER L.L. \& BocK I.R. 1972: Isozyme variation and phylogenetic relationship in the Drosophila bipectinata species complex. Univ. Texas Publ. 7213: 213-227.

ZAR J.H. 2005: Biostatistical Analysis. 4th ed. Pearson Education, Delhi, $736 \mathrm{pp}$.

Received October 5, 2005; revised and accepted April 13, 2006 